\title{
Cognitive reserve in the healthy elderly: cognitive and psychological factors
}

\author{
Josef Zihl ${ }^{1,2 *}$, Antonia Schmid ${ }^{1}$, Osborne F.X. Almeida ${ }^{2}$, Nuno Sousa ${ }^{3}$, Katrin Walther ${ }^{1}$, and Thomas Fink ${ }^{1}$ \\ ${ }^{1}$ Department Psychology, Neuropsychology, Lud1wig-Maximilians-Universität München, Munich, Germany \\ ${ }^{2}$ Max Planck Institute of Psychiatry, Munich, Germany \\ ${ }^{3}$ Life and Health Sciences Research Institute (ICVS), School of Health Sciences, University of Minho, Braga, Portugal, and \\ ICVS/3B's, PT Government Associate Laboratory, 4710-057 Braga/Guimarães, Portugal \\ *Corresponding author's e-mail address: zihl@psy.Imu.de
}

Published online: September 29, 2014 (version 1)

Cite as: Zihl et al., ScienceOpen Research 2014 (DOI: 10.14293/S2199-1006.1.SOR-SOCSCI.ADKHNX.v1)

Reviewing status: Please note that this article is under continuous review. For the current reviewing status and the latest referee's comments please click here or scan the QR code at the end of this article.

Primary discipline: Psychology

Keywords: Mental Aging, Cognitive Reserve, Cognitive Architecture

\begin{abstract}
Cognitive reserve (CR) helps explain the mismatch between expected cognitive decline and observed maintenance of cognitive functioning in older age. Factors such as education, literacy, lifestyle, and social networking are usually considered to be proxies of $\mathrm{CR}$ and its variability between individuals. A more direct approach to examine CR is through the assessment of capacity to gain from practice in a standardized challenging cognitive task that demands activation of cognitive resources. In this study, we applied a testing-the-limits paradigm to a group of 136 healthy elderly subjects (60-75 years) and additionally examined the possible contribution of complex mental activities and quality of sleep to cognitive performance gain. We found a significant but variable gain and identified verbal memory, cognitive flexibility, and problemsolving as significant factors. This outcome is in line with our earlier study on CR in healthy mental aging. Interestingly and contrary to expectations, our analysis revealed that complex mental activities and sleep quality do not significantly influence CR. Contrasting "high" and "low" cognitive performers revealed significant differences in verbal memory and cognitive flexibility; again, complex mental activities and sleep quality did not contribute to this measure of CR. In conclusion, the results of this study support and extend previous findings on CR in older age; further, they underline the need for improvements in existing protocols for assessing CR in a dynamic manner.
\end{abstract}

\section{INTRODUCTION}

The concept of cognitive reserve (CR) attempts to explain interindividual variability in susceptibility to changes in brain function in pathological, but also normal aging of the brain $[1,2]$. In their model of CR, Satz et al. [3] proposed four factors (general intelligence ("g"), complex mental activity, processing resources, and executive function) as "potential reserve proxies" for CR in normal aging. Each of these factors encompasses one of several specific indicators that contribute to, or interact with, CR. Examples of such factors include literacy and education, occupation, regular complex mental activities, and cohesiveness of social networks [4-8]. It is also plausible that other variables, such as mood [9] and quality of sleep [10] may also determine cognitive performance in older age. In addition, it should be noted that many of these putative CR-determining factors may be intercorrelated [11], and that they are influenced by individual life experiences and thus, by developmental trajectories in early adulthood and middle age [12].

Since CR (in the following, we use the abbreviation CR to denote gains in the testing-the-limits paradigm, which serve as "intimate" proxies of CR) cannot be measured directly, the assessment of proxies is valuable; however, these proxies are likely to be static rather than dynamic representations of CR. From a conceptual and methodological perspective, it seems desirable to define and assess CR in a more direct fashion, e.g., by "forcing" subjects to activate their individual cognitive resources in a mentally challenging task. Such an approach would also fit into a model of task-related brain activity depending on the level of task demands, which helps to explain interindividual differences in the context of brain reserve and thus of the neural basis of CR [13]. Based on this framework, one would predict that subjects with higher functional brain reserve would also show higher performance in such a task because they can activate more cognitive resources in this condition. The gain in performance may thus represent a more valid proxy of CR because it is based on the dynamic use of implicitly existing CR, which cannot be determined validly in a one-trial assessment. A particularly sensitive method to assess CR in this framework is the 
so-called testing-the-limits paradigm [14]. The use of the Digit Symbol Substitution Test (DSST), a multi-tasking cognitive measure [15], in a testing-the-limits paradigm with systematic repetition offers a way to assess CR in a dynamic way. In our recent study that contrasted 140 younger with 140 older healthy subjects, all of whom had a similar level of education [16], we used such a paradigm to assess CR. The main outcome of that study was that systematic practice with DSST leads to significant gains in both age groups. Interestingly, although CR was significantly higher in the younger subjects, about $50 \%$ of older subjects showed similar CR values, suggesting that CR may, at least partly, represent an individual, age-independent factor. As predicted from the model of Satz et al. [3], CR was significantly associated with speed of information processing, verbal working memory, and problem-solving in the older group; no such associations were found in the younger group.

Because DSST as a testing-the-limits-paradigm has only been used in a few studies on mental aging research, and reproducibility is a "cornerstone of science" [17], we considered it important to demonstrate that this instrument is a valid and reliable measure of $\mathrm{CR}$. In the current study, we therefore assessed CR with the same testing-the-limits paradigm in 136 healthy elderly subjects (age range: 60-75 years), all of whom had a similar high level of education $(\geq 13$ years of schooling). In addition, we examined the role of diverse cognitive and psychological variables in CR, i.e., cognitive architecture, mood, cognitive, social and physical activities, sleep quality, and well-being (quality of life, life satisfaction). According to the model of Satz et al. [3], complex mental activity, processing resources, and executive function represent core factors of CR. Complex mental activities ("cognitive lifestyle") represent a significant factor for CR because they are associated with more favorable cognitive trajectories in older persons $[4,6]$ and may even support reversal from mild cognitive impairment (MCI) to normal cognitive functioning [18]. Accordingly, our main hypotheses were that (1) subjects with higher processing resources and higher executive capacities will show higher CR, and (2) CR is positively correlated with cognitive lifestyles, mood, well-being, and sleep quality. To further elucidate the influence of these factors on CR, we contrasted high and low performers using an extreme-group comparison analysis.

\section{MATERIALS AND METHODS Participants}

A total of 138 healthy elderly subjects aged between 60 and 75 years with at least 13 years of education (range: 13-21 years) participated in this study. Older subjects were recruited from senior university students enrolled at LudwigMaximilians-Universität (Munich); their relatives and friends served as further participants. Before admittance to the study, subjects were given a detailed telephone interview to screen for exclusion criteria, in particular neurological and mental disorders as well as non-correctable visual or auditory impairments and motor limitations of the dominant (right or left) hand. According to the Edinburgh handedness inventory [19], 97\% of subjects were right-handed, three were lefthanded, and three were ambidextrous. Two subjects were excluded due to incomplete datasets; thus, 136 participants (68 female, 68 male; age: $M=68.71$ years, $S D=3.60$ years) were studied in detail.

Each participant was tested with the Mini-Mental State Examination (MMSE of the CERAD-Plus; [20]) to exclude subjects with global cognitive impairment (MMSE $\leq 26$ points). In addition, detailed information on education and occupation, medical history, medication, smoking, and alcohol use was collected.

\section{Assessment of cognitive and psychological variables}

Cognitive measures: Cognitive architecture was assessed using standard one-time measurements in the following cognitive domains: cognitive multitasking (DSST; German version; [21], number of correct symbols), verbal learning and long-term memory (word list memory and recall of the CERAD-Plus; [20], number of correctly recalled items in 3 consecutive trials and after delay), verbal short-term and working memory (Digit Span forward and backward; WAIS-III, German version; [21], number of correctly recalled digits), information processing speed/attention and cognitive flexibility (Trail Making Test, TMT, Parts A and B, of the CERAD-Plus; [20], response time), interference (Stroop Color and Word Test, German Version; [22], hits per 45 s for word and colour conditions and for interference condition), verbal fluency (Regensburger Word Fluency Test, RWT; [23], total number of correctly produced words for letters S, K, and M, within $1 \mathrm{~min}$ ), visual problem-solving (Matrix Reasoning of the WAIS-III, German version; [21], number of correct items), and reading performance (standard text of 200 words, measured in words per minute, wpm).

Psychological measures: The above-mentioned cognitive tests were complemented by assessments of mood (Geriatric Depression Scale, GDS, a 15-items short form, German version; [24]), sleep quality (Pittsburgh Sleep Quality Index, PSQI; German translation; [25]), quality of life (World Health Organization Quality of Life assessment instrument, short form, WHOQOL-BREF, German version; [26]), and life satisfaction (Satisfaction with Life Scale, SWLS; German translation; [27]), as well as leisure activities (self-generated questionnaire of physical, social, and cognitive activities).

Measurement of CR: To determine improvement after systematic practice, a modified version of the DSST was used. In the original DSST, subjects are required to assign nine different symbols to the digits "1-9" based on a previously introduced combination. The modified test was identical to the original DSST, except that different symbols were introduced which subjects had to match to digits. In the testing-the-limitsparadigm, participants were required to fill in the missing symbols with a pencil as fast and accurately as possible in 
each of 10 consecutive trials (30-second inter-trial intervals were used to avoid motor fatigue of the hand). To avoid ceiling effects, the time for the trials was limited to $90 \mathrm{~s}$ (standard: $120 \mathrm{~s}$ ). The number of correctly assigned symbols was used as a measure of performance. Test-retest reliability of the DSST is known to be high (0.82-0.84; [28]), indicating uniformity of changes in performance. Further, performance in the DSST is sufficiently sensitive to mental aging and is independent of years of education [29].

\section{Procedure}

All test sessions were carried out between 9 am and $1 \mathrm{pm}$ and, including adequate breaks, lasted approximately three hours. Tests were administered by three well-trained psychologists.

The study design conformed to the principles outlined in the latest version of the Declaration of Helsinki and was approved by the Ethical Committee of the Medical Faculty of LudwigMaximilians-Universität. Written informed consent was provided by each participant in the study; participants received a small financial compensation (€30).

\section{Data analysis}

Calculation of indexes of CR: Given the usefulness of simple gain scores [30], we used the following formula as an index for CR [16]:

$$
\begin{aligned}
& \qquad \mathrm{CR}=\sum_{i=1}^{10}\left(1+\frac{x_{1}}{x_{\max }}\right) \times \frac{x_{i}-x_{1}}{x_{\max }} \\
& x_{1}=\text { baseline, } x_{\max }=\text { population maximum, and } \\
& x_{i}=\text { single trial. }
\end{aligned}
$$

where the gain in every trial is divided by the population maximum and multiplied by the quotient of the baseline and population maximum. The sum of these products provides the area under the curve of the relativized gain function. This product is set at 0 in the first trial and may increase (positive values) or decrease (negative values) with relative increments or decrements in DSST performance during successive trials. Statistical analysis of data: Data were analyzed using IBM SPSS Statistics 20. Pure performance gain, i.e., difference between correctly assigned symbols in the first and in the best trial of the 10 consecutive trials, was calculated with a dependent samples $t$-test of maximum vs. initial score in the modified DSST. Relationships within measures of cognitive architecture and psychological factors, and between these measures and gain scores, were analyzed using bivariate correlation analysis. This analysis was also used to test relationships between age and CR, and cognitive as well as psychological variables. Differences between types of leisure activities were analyzed using a repeated-measures analysis of variance(ANOVA). In addition, differences in gain scores, cognitive architecture, and psychological factors were tested with respect to gender by applying independent samples $t$-tests. In a more detailed analysis using extreme group comparisons [31], subjects' CR was classified as "high" and "low", based on whether individual gain scores fell within the $25 \%$ uppermost or lowermost values of the total group gain. Differences in cognitive and psychological variables between the high and low performers were compared by means of independent samples $t$-tests. All corresponding $p$-values reported are Bonferroni-corrected due to multiple comparisons, with significance level set at $\alpha=.05$.

\section{RESULTS}

\section{Cognitive and psychological measures}

Cognitive architecture: Outcomes of the various subtests are shown in Table 1. For those tests where standardized

\begin{tabular}{|c|c|c|c|c|}
\hline \multirow[b]{2}{*}{ Cognitive architecture } & \multicolumn{2}{|c|}{ Descriptives } & \multicolumn{2}{|c|}{ Correlation with $\mathrm{CR}$} \\
\hline & $M$ & $S D$ & $r$ & $p$ \\
\hline DSST & 67.20 & 14.01 & .13 & .96 \\
\hline WL Learning & 22.89 & 3.29 & $.32^{*}$ & $<.01$ \\
\hline WL Delay & 7.97 & 1.65 & $.35^{*}$ & $<.01$ \\
\hline DS forwards & 5.76 & 1.04 & .14 & .74 \\
\hline DS backwards & 4.48 & 1.07 & .19 & .21 \\
\hline TMT A & 39.19 & 12.50 & .06 & .99 \\
\hline TMT B-A & 44.40 & 21.45 & $-.31^{*}$ & $<.01$ \\
\hline \multicolumn{5}{|l|}{ Stroop } \\
\hline Words & 100.45 & 13.43 & .09 & .99 \\
\hline Colors & 69.02 & 10.40 & .18 & .23 \\
\hline interference & 41.22 & 7.50 & .22 & .07 \\
\hline RWT & 45.10 & 9.80 & .20 & .12 \\
\hline MR & 18.89 & 4.58 & $.28^{*}$ & $<.01$ \\
\hline \multirow[t]{2}{*}{ Reading } & 169.16 & 26.83 & .17 & .34 \\
\hline & \multicolumn{2}{|c|}{ Descriptives } & \multicolumn{2}{|c|}{ Correlation with $\mathrm{CR}$} \\
\hline Psychological factors & $M$ & $S D$ & $r$ & $p$ \\
\hline GDS & 1.31 & 1.61 & -.12 & .98 \\
\hline PSQI & 5.01 & 3.05 & -.10 & .99 \\
\hline \multicolumn{5}{|l|}{ WHOQOL-BREF: } \\
\hline Total & 79.41 & 11.54 & .10 & .99 \\
\hline Physical & 83.43 & 11.77 & .02 & .99 \\
\hline psychological & 77.17 & 11.46 & .12 & .99 \\
\hline Social & 70.68 & 16.22 & -.06 & .99 \\
\hline environmental & 88.08 & 9.73 & .12 & .99 \\
\hline SWLS & 12.80 & 4.55 & .01 & .99 \\
\hline \multicolumn{5}{|l|}{ Leisure activities: } \\
\hline Physical & 31.38 & 9.31 & $<.01$ & .99 \\
\hline Social & 34.82 & 7.75 & -.10 & .99 \\
\hline Cognitive & 49.71 & 3.05 & -.05 & .99 \\
\hline
\end{tabular}

Table 1. Cognitive architecture and psychological factor outcomes and their correlation with $\mathrm{CR}$.

Note: DSST, digit symbol substitution test; WL, CERAD-Plus word list; DS, digit span; TMT, trail making test; Stroop, Stroop color and word test; RWT, Regensburger world fluency test; MR, matrix reasoning; GDS, geriatric depression scale; PSQI, Pittsburgh sleep quality index; WHOQOL-BREF, WHO quality of life (short form); SWLS, satisfaction with life scale.

${ }^{*} p<.01$, 1-tailed. 
comparison values were available, the results of our sample lie within the normal range (e.g. CERAD-Plus word list, Digit Spans of WAIS-III, TMT, DSST, RWT) or above average (Matrix Reasoning of WAIS-III). Females showed significantly higher scores in verbal learning (CERAD-Plus word list, learning trials: $t(134)=3.21, p=.03, d=.55)$ and memory (CERAD-Plus word list, delay: $t(126.02)=3.69, p<.01, d=$ .64) compared to male subjects. Not surprisingly, we found high intercorrelations between subtests of cognitive architecture, with DSST having a moderate influence on all other measures (lowest correlation with CERAD-Plus word list, delay: $r=.24, p=.06$; highest correlation with TMT [time B-time A] as measure of cognitive flexibility: $r=-.44, p<$ .01). Reading performance revealed moderate-to-high correlations with Stroop color and word subtests ( $r$ between .35 and $.58, p<.01$, respectively), and further significant correlations with verbal fluency RWT $(r=.32, p<.01)$ as well as DSST $(r=.27, p=.01)$; however, these measures did not correlate significantly with verbal memory measures, information processing speed, and cognitive flexibility (largest $r=.23$, $p=.10)$.

Psychological factors: Table 1 shows subjects' responses to questionnaire-based assessment of psychological variables. Overall, subjects displayed low scores on GDS (maximum range: $0-15$, cut-off $>6$ ) and SWLS (maximum range: 7-35), and high scores on all WHOQOL-BREF subscales (maximum = 100). Sleep quality (PSQI - total score) was of average quality $(M=5.01, S D=3.05)$. With respect to leisure engagement, subjects demonstrated significantly higher engagement in cognitive vs. physical and social activities, $F(1.86,248.78)=$ 221.68, $p<.001, \eta_{\mathrm{p}}^{2}=.62$. There was no evidence that psychological measures were influenced by aging (highest correlation with GDS: $r=.18, p=.40$ ). Males only differed from females in terms of mental aspects of life quality (psychological subscale of WHOQOL-BREF: $t(134)=3.68, p<.01, d=$ $.64)$, with scores being higher in males $(M=80.63, S D=9.99)$ than females $(M=73.71, S D=11.85)$. Numerous correlations were found between psychological factors; in particular, mood (GDS) was related to most other domains, with relatively uniform moderate-to-strong effect sizes (lowest correlation with physical activity: $r=-.21, p=.16$; highest correlation with psychological score of WHOQOL-BREF: $r=-.62$, $p<.01$ ).

Relationship between cognitive architecture and psychological factors: Correlations between measures in subtests of cognitive architecture for cognitive multitasking (DSST), verbal memory (word list delay, CERAD-Plus), information processing speed/attention (TMT A), cognitive flexibility (TMT B-A), visual problem-solving (Matrix Reasoning), and reading performance, with mood (GDS), sleep quality (PSQI), and leisure activities (combined) are shown in Table 2. Significant correlations with weak-to-moderate effect sizes were only found between mood and information processing speed/attention $(r=.27, p=.01)$, and between extent of leisure activities and
Table 2. Correlations between measures of cognitive architecture and psychological factors.

\begin{tabular}{|c|c|c|c|c|c|c|}
\hline \multirow[b]{3}{*}{ Cognitive architecture } & \multicolumn{6}{|c|}{ Psychological factors } \\
\hline & \multicolumn{2}{|c|}{ GDS } & \multicolumn{2}{|c|}{ PSQI } & \multicolumn{2}{|c|}{ Activities } \\
\hline & $r$ & $p$ & $r$ & $p$ & $r$ & $p$ \\
\hline DSST & -.19 & .99 & -.08 & .99 & $.23 *$ & .05 \\
\hline WL Delay & .03 & .99 & .10 & .99 & .04 & .99 \\
\hline TMT A & $.27^{* *}$ & .01 & .09 & .99 & -.18 & .20 \\
\hline TMT B-A & .02 & .99 & .10 & .99 & -.13 & .73 \\
\hline MR & -.07 & .99 & .06 & .99 & .18 & .23 \\
\hline Reading & .06 & .99 & .04 & .99 & .04 & .99 \\
\hline
\end{tabular}

Note: DSST, digit symbol substitution test; WL, CERAD-Plus word list; TMT, trail making test; MR, matrix reasoning; GDS, geriatric depression scale; PSQI, Pittsburgh sleep quality index.

${ }^{*} p \leq .05$, two-tailed. ${ }^{* *} p \leq .01$, two-tailed.

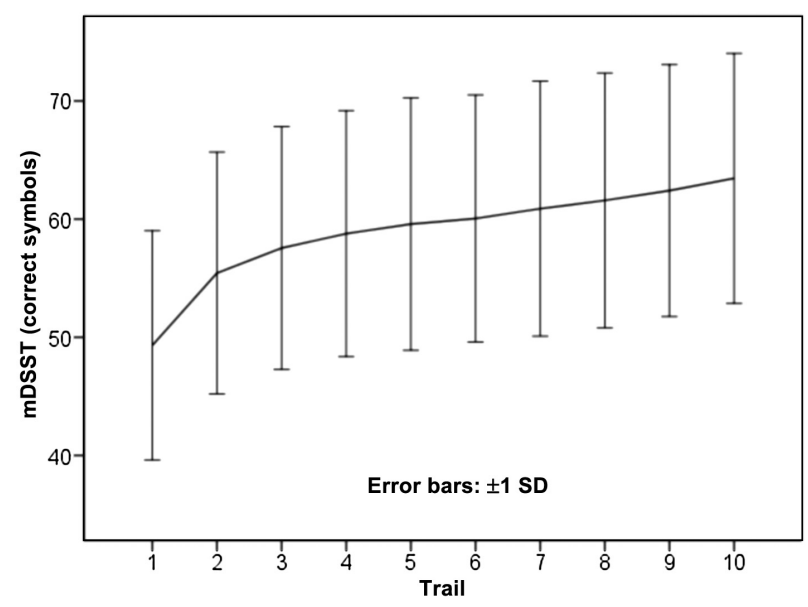

Figure 1. Mean score in the modified DSST in 10 consecutive trials of the testing-the-limits paradigm for the assessment of CR. Vertical bars indicate \pm 1 SD.

cognitive multitasking ( $r=.23, p=.05)$. No significant correlation was found between reading performance and any psychological factor (largest correlation with WHOQOL-BREF, total score: $r=.13, p=.99$ ).

\section{Cognitive reserve}

Analysis of the effect of systematic practice on the modified DSST (10 trials) is illustrated in Figure 1. Participants correctly assigned an average of $49.32(S D=9.70)$ symbols in the first trial and showed a 15-item improvement in performance in the last trial $(M=64.85, S D=10.73)$. Despite the large variability in performance, the increase in performance was highly significant $(t(135)=30.06, p<.001, d=1.52)$. There were no significant differences in gain scores between 
males and females $(t(134)=.56, p=.58, d=.10)$. Moreover, gain scores did not correlate with age $(r=-.09, p=.32)$.

Relationship between cognitive architecture and CR: Results of correlational analyses between gain scores and domains of cognitive architecture are also displayed in Table 1. Significant correlations with moderate effect sizes were only found for verbal learning (CERAD-Plus word list, learning trials: $r=.32, p<.01$ ) and verbal memory (CERAD-Plus wordlist, delay: $r=.35, p<.01$ ), cognitive flexibility (TMT, B-A: $r=-.31, p<.01$ ), and visual problem-solving (Matrix Reasoning, WAIS-III: $r=.28, p<.01$ ). There was a positive trend between $\mathrm{CR}$ and inhibition (Stroop test, interference condition: $r=.22, p=.07)$. Interestingly, no significant correlation was found between $\mathrm{CR}$ and reading performance $(r=.17, p=.34)$.

Relationship between $C R$ and psychological factors: Furthermore, as can be seen in Table 1, correlations between gain scores and psychological measures failed to reach significance. Coefficients indicate only weak (e.g. GDS, PSQI) or negligible interactions (e.g. SWLS, physical engagement) between gain and subjective well-being.

Differences between high and low performers in cognitive architecture and psychological factors: To test for differences between high and low performers, gain scores were separated into the upper and lower quartiles of the distribution, respectively. The mean CR was $2.48(S D=.44)$ in high performers and $.73(S D=.23)$ in lower performers, displaying a highly significant difference $(t(49.68)=20.47, p<.001$, $d=5.04)$. Cognitive architecture differed significantly in terms of verbal learning (CERAD-Plus word list: $t(58.55)=$ 3.69, $p<.01, d=.91$ ), verbal memory (CERAD-Plus word list, delay: $t(50.05)=4.02, p<.01, d=.98)$, and cognitive flexibility (TMT B-A: $t(59.42)=3.63, p=.01, d=.89$ ). Reading performance differed by 15 words per minute between high and low performers, albeit non-significantly $(t(66)=2.14, p=.47$, $d=.53$ ). No significant differences were found in psychological measures obtained from low and high performers (largest difference for sleep quality, PSQI: $t(66)=1.87$, $p=.72, d=.46$ ).

\section{DISCUSSION}

A major finding to emerge from this study is that repeated practice by healthy subjects, aged between 60 and 75 years, can result in considerable improved performance in a complex cognitive task such as the DSST; this was seen despite considerable within-group variation in CR. The improvements were not confined to one gender and did not depend on age (in the range examined). Importantly, significant correlations between measures of cognitive architecture and CR were found for verbal memory, cognitive flexibility, and visual problem-solving, whereas psychological variables (e.g. mood, sleep quality, quality of life, life satisfaction, and leisure activities) did not significantly correlate with CR. Notably, complex mental activity and sleep quality did not play a significant role in CR; this finding was unpredicted. An extreme group comparison method revealed significantly better verbal learning, verbal memory, and cognitive flexibility in high vs. low performers. However, high performers did not differ significantly from low performers in terms of complex mental activities and sleep quality or any of the psychological measures.

Overall, the present results are consistent with those of our previous study [16] and favor the evidence that activation of cognitive resources is well preserved in the healthy elderly, notwithstanding individual differences in performance gains. In this study, performance gain in the DSST testing-the-limits paradigm was 15.53 items $(S D=6.03)$, as compared to 18.94 items $(S D=7.30)$ in our earlier study [16]. Interestingly, the younger group (20-30 years) in our earlier study [16] showed a higher gain (25.52), but variability was in a similar range $(S D=10.10)$. This supports the idea that $C R$ is not a quality of older age alone [32], and that interindividual variability is already apparent at earlier ages; therefore, aging is not the main or only determinant of CR variability in older age. In addition, we found a significant association between $\mathrm{CR}$, verbal memory, and executive function, but no association between $\mathrm{CR}$ and gender, in both studies. Apart from three measures of cognitive architecture (verbal learning, verbal memory, and cognitive flexibility), high performers did not differ significantly from low performers, indicating that CR may depend on particular, rather than general, components of cognitive architecture. It is likely that this interpretation may not apply to other dynamic measures of CR in which other components of cognitive architecture may be a prerequisite for achieving high gain. Nevertheless, the positive relationship between verbal learning, verbal memory, executive function, and CR indicates that these cognitive capacities play a crucial role in determining performance in the DSST. Roldán-Tapia et al. [33] found a significant contribution of speed of information processing, verbal memory, and executive function to CR (defined as a proxy for verbal intelligence, educational level, and profession). Notably, Santos et al. [9] reported memory and executive function as the main cognitive dimensions that separate high and low performers in cognitive architecture. Together, these results support the idea of a "central circuit of the mind" in which brain structures underlying declarative memory and executive function that serve as basic modules of cognitive architecture [34].

In contrast to earlier studies [35, 36], we did not find evidence that years of education (educational level) and reading performance play an essential role for CR in our group. This discrepancy is possibly explained by the fact that all subjects in the present study had an above-average (high) level of education and reported high cognitive engagement in leisure activities as well as regular reading. As Reed et al. [37], and Kavé et al. [38] have shown, cognitive leisure activities and regular reading are more important than level of formal education for later cognitive functioning and CR proxies. This 
view is supported by the finding that, although education is related to CR, it does not necessarily slow cognitive decline in older age [39], while active cognitive lifestyle does [4, 6]. Interestingly, this also holds true for premorbid cognitive activities and CR proxies in subjects with multiple sclerosis [40] and MCI [18, 41].

Mood and sleep quality of our subjects did not have significant effects on their CR; this finding is not surprising given that all subjects in this study reported good mood and sleep quality. Murphy \& 0'Leary [42] found an association between depressive symptoms and poor verbal memory, with higher education levels contributing to higher performance. Zimmermann et al. [43] reported that only older adults with lower education suffer from the negative effects of difficulties in sleep onset and maintenance on CR. Thus, pathological states of mood and sleep quality may have a significant impact on cognitive architecture; higher education may serve as a protective factor in both cases. Interestingly, however, comparison of high and low performers in this study revealed higher performance in measures of cognitive architecture in subjects with higher mood scores and higher engagement in leisure activities, supporting the view that mood and cognitive lifestyle are key determinants of cognitive performance in healthy older individuals $([6,9,18])$.

The present data contribute to the CR model proposed by Satz et al. [3] by confirming the assumption that processing resources, verbal memory, executive function, and complex cognitive activities promote CR. Interestingly, despite narrow selection criteria, the subjects in this study showed high variability in CR, indicating that CR may be influenced strongly by subject-specific characteristics. This question must be addressed in future tests of the CR model proposed by Satz et al. [3], where CR is assessed in subgroups of healthy older subjects by considering demographic variables, educational level, cognitive lifestyle, including reading and mood. Investigations of large groups will help to reveal the effect of these variables on a large continuum. Although such studies will not permit unequivocal conclusions about their specific role(s) on CR, they will help better define more valid "proxies". Individual differences in CR may mirror individual differences in the interplay between genetics and environment and thus, brain function (phenotypes; [44]). Furthermore, biological variables, such as hormonal profiles [45], brain morphology [46], and individual resting state activity [47], may potentially contribute to interindividual differences in "cognitive plasticity" and thus, CR. However, part of the differences in the high and low performers may be attributed to age-independent interindividual differences in cognition and cognitive plasticity [32]; the outcome of our earlier study [16] would also support such an assumption.

In conclusion, the results of this study support and extend the model proposed by Satz et al. [3] and suggest the need for a more dynamic standardized assessment of CR; such assessment should consider that "cognitive reserve is not fixed but continues to evolve across life span" [48]. Our results confirm the validity of measures of gain in performance in a cognitively challenging testing-the-limits paradigm as an essential measure of CR in healthy mental aging; such an approach aligns with the theoretical research model proposed by Steffener \& Stern [13], which attempts to unify the notion of neural vs. CR. In addition, this paradigm may be also helpful in the differential diagnostic assessment of potentially existent vs. absent cognitive resources, especially in subjects with suspected or proven cognitive impairments.

\section{ACKNOWLEDGMENTS}

This work was funded by the European Commission (FP7) "Switchbox" (Contract HEALTH-F2-2010.259772). We are thankful to all participants. We would like to thank Ruth von Hammerstein for assistance with data collection and Karin Münzel for organizational help with the study.

\section{REFERENCES}

[1] Satz P. Brain reserve capacity on symptom onset after brain injury: a formulation and review of evidence for threshold theory. Neuropsychology. 1993;7:273-95. doi:10.1037/0894-4105. 7.3.273

[2] Stern Y. Cognitive reserve. Neuropsychologia. 2009;47:2015-28. doi:10.1016/j.neuropsychologia.2009.03.004

[3] Satz P, Cole MA, Hardy DJ, Rassovsky Y. Brain and cognitive reserve: mediator (s) and construct validity, a critique. J Clin Exp Neuropsychol. 2011;33: 121-30. doi:10.1080/13803395. 2010.493151

[4] Hall CB, Lipton RB, Sliwinski M, Katz MJ, Derby CA, Verghese J. Cognitive activities delay onset of memory decline in persons who develop dementia. Neurology. 2009;73:356-61. doi:10.1212/ WNL.0b013e3181b04ae3

[5] Foubert-Samier A, Catheline G, Amieva H, Dilharreguy B, Helmer $C$, et al. Education, occupation, leisure activities, and brain reserve: a population-based study. Neurobiol Aging. 2012;33: 423.e15-423.e25. doi:10.1016/j.neurobiolaging.2010.09.023

[6] Marioni RE, van den Hout A, Valenzuela MJ, Brayne C, Matthews FE. Active cognitive lifestyle associates with cognitive recovery and a reduced risk of cognitive decline. J Alzheimers Dis. 2012; 28:223-30. doi:10.3233/JAD-2011-110377

[7] Small BJ, Dixon RA, McArdle JJ, Grimm KJ. Do changes in lifestyle engagement moderate cognitive decline in normal aging? Evidence from the Victoria Longitudinal study. Neuropsychology. 2012;26:144-55. doi:10.1037/a0026579

[8] Valenzuela M, Sachdev P. Can cognitive exercise prevent the onset of dementia? Systematic review of randomized clinical trials with longitudinal follow-up. Am J Geriatr Psychiatry. 2009;17:179-87. doi:10.1097/JGP.0b013e3181953b57

[9] Santos NC, Costa PS, Cunha P, Cotter P, Sampaio A, et al. Mood is a key determinant of cognitive performance in communitydwelling older adults: a cross-sectional analysis. Age (Dordr). 2013;35:1983-93. doi:10.1007/s11357-012-9482-y

[10] Nebes RD, Buysse DJ, Halligan EM, Houck PR, Monk TH. Selfreported sleep quality predicts poor cognitive performance in healthy older subjects. J Gerontol B Psychol Sci Soc Sci. 2009;64:180-7. doi:10.1093/geronb/gbn037

[11] Ceci SJ. How much does schooling influence general intelligence and its cognitive components? Dev Psychol. 1991;27:703-22. doi:10.1037/0012-1649.27.5.703 
[12] Richards M, Sacker A. Lifetime antecedents of cognitive reserve. J Clin Exp Neuropsychol. 2003;25:614-24. doi:10.1076/jcen.25. 5.614 .14581

[13] Steffener J, Stern Y. Exploring the neural basis of cognitive reserve in aging. Biochimica et Biophysica Acta. 2012;1822: 467-73. doi:10.1016/j.bbadis.2011.09.012

[14] Baltes MM, Kühl K-P, Sowarka D. Testing the limits of cognitive reserve capacity: a promising strategy for early diagnosis of dementia? J Gerontol, 1992;47:165-7. doi:10.1093/geronj/47.3. P165

[15] Lezak MD, Howieson DB, Loring DW. Neuropsychological assessment. Oxford: Oxford University Press; 2004.

[16] Zihl J, Fink T, Pargent F, Ziegler M, Bühner M. Cognitive reserve in young and old healthy subjects: differences and similarities in a testing-the limits paradigm with DSST. PLoS One. 2014;9: e84590. doi:10.1371/journal.pone.0084590.t003

[17] Simons DJ. The value of direct replication. Psychol Sci. 2014;9:76-80. doi:10.1177/1745691613514755

[18] Sachdev PS, Lipnicki DM, Crawford J, Reppermund S, Kochan NA, et al. Factors predicting reversion from mild cognitive impairment to normal cognitive functioning: a population-based study. PLoS One. 2013;8:e59649. doi:10.1371/journal.pone.005 9649.s003

[19] Oldfield RC. The assessment and analysis of handedness: the Edinburgh inventory. Neuropsychologia. 1971;9:97-113. doi:10.1016/0028-3932(71)90067-4

[20] Memory Clinic Basel. CERAD-Plus: the consortium to establish a registry for Alzheimer's disease. Basel, Switzerland: Universitätsspital; 2005.

[21] Aster MV, Neubauer A, Horn R., Eds. WAIS-III. Wechsler adult intelligence scale [German version]. Frankfurt/M, Germany: Harcourt; 2006.

[22] Bäumler G. Stroop color and word test [German version]. Göttingen, Germany: Hogrefe; 1985.

[23] Aschenbrenner S, Tucha O, Lange KW. Regensburger word fluency test. Göttingen, Germany: Hogrefe; 2000.

[24] Gauggel S, Birkner B. Validity and reliability of a German version of the Geriatric Depression Scale (GDS). Zeitschrift für Klinische Psychologie. 1999;28:18-27. doi:10.1026//00845345.28.1.18

[25] Buysse DJ, Reynolds III CF, Monk TH, Berman SR, Kupfer DJ. The Pittsburgh sleep quality index: a new instrument for psychiatric practice and research. Psychiatry Res. 1989;28:193213. doi:10.1016/0165-1781(89)90047-4

[26] Angermeyer MC, Kilian R, Matschinger H. WHOQOL-100 and WHOQOL-BREF: Handbook of the German version of the WHO instruments for the assessment of life quality. Göttingen: Hogrefe; 2000.

[27] Diener ED, Emmons RA, Larsen RJ, Griffin S. The satisfaction with life scale. J Pers Assess. 1985;49:71-5. doi:10.1207/s153 27752jpa4901_13

[28] Matarazzo JD, Herman DO. Base rate data for the WAIS-R: testretest stability and VIQ-PIQ differences. J Clin Neuropsychol. 1984;6: 351-66. doi:10.1080/01688638408401227

[29] Hoyer WJ, Stawski RS, Wasylyshyn C, Verhaeghen P. Adult age and digit symbol substitution performance: a meta-analysis. Psychol Aging. 2004;19:211. doi:10.1037/0882-7974.19.1.211

[30] Williams RH, Zimmerman DW. Are simple gain scores obsolete? Appl Psychol Meas. 1996;20:59-69. doi:10.1177/0146621696 02000106

[31] Preacher KJ, Rucker DD, MacCallum RC, Nicewander WA. Use of the extreme groups approach: a critical reexamination and new recommendations. Psychol Methods. 2005;10:178-92. doi:10.1037/1082-989X.10.2.178

[32] Salthouse TA. Does the level at which cognitive change occurs change with age? Psychol Sci. 2012;23:18-23. doi:10.1177/ 0956797611421615

[33] Roldán-Tapia L, Garcia J, Cánovas R, León I. Cognitive reserve, age, and their relation to attentional and executive functions. Appl Neuropsychol. 2012;19:2-8.

[34] Anderson JR, Fincham JM, Quin Y, Stocco A. A central circuit of the mind. Trends Cogn Sci. 2008;12:136-43. doi:10.1016/j. tics.2008.01.006

[35] Jefferson AL, Gibbons LE, Renz DM, Carvalho JO, Manly J, et al. A life course model of cognitive activities, socioeconomic status, education, reading ability, and cognition. J Am Geriatr Soc. 2011;59:1403-11. doi:10.1111/j.1532-5415.2011.03499.x

[36] Paulo AC, Sampaio A, Santos NC, Costa PS, Cunha P, et al. Patterns of cognitive performance in healthy ageing in Northern Portugal: a cross-sectional analysis. PLoS One. 2011;6:e24553. doi:10.1371/journal.pone.0024553.t003

[37] Reed BR, Dowling M, Farias ST, Sonnen J, Strauss M, et al Cognitive activities during adulthood are more important than education in building reserve. J Int Neuropsychol Soc. 2011; 17:615-24. doi:10.1017/S1355617711000014

[38] Kavé G, Shrira A, Palgi Y, Spalter T, Ben-Ezra M, et al. Formal education level versus self-rated literacy as predictors of cognitive aging. J Gerontol B Psychol Sci Soc Sci. 2012;67:697-704. doi:10.1093/geronb/gbs031

[39] Zahodne LB, Glymour MM, Sparks C, Bontempo M, Dixon RA, et al. Education does not slow cognitive decline with aging: 12-year evidence from the Victoria Longitudinal Study. J Int Neuropsychol Soc. 2011;17:1039-46. doi:10.1017/S13556177 11001044

[40] Sumowski JF, Rocca MA, Leavitt VM, Dackovic J, Mesaros S, Drulovic J, DeLuca J, Philippi M. Brain reserve and cognitive reserve protect against decline over 4.5 years in MS. Neurology. 2014;82:1776-83. doi:10.1212/WNL.0000000000000433

[41] Liu Y, Cai ZL, Xue S, Zhou X, Wu F. Proxies of cognitive reserve and their effects on neuropsychological performance in patients with mild cognitive impairment. J Clin Neurosci. 2013;20:54853. doi:10.1016/j.jocn.2012.04.020

[42] Murphy M, O'Leary E. Depression, cognitive reserve and memory performance in older adults. Int J Geriatr Psychiatry. 2010;25:665-71. doi:10.1002/gps.2404

[43] Zimmermann ME, Bigal ME, Katz MJ, Brickman AM, Lipton RB. Sleep onset/maintenance difficulties and cognitive function in nondemented older adults: the role of cognitive reserve. J Int Neuropsychol Soc. 2012;18:461-70. doi:10.1017/S1355617711 001901

[44] Deary IJ. Intelligence. Annu Rev Psychol. 2012;63:453-82. doi:10.1146/annurev-psych-120710-100353

[45] Auyeung B, Lombardo MV, Baron-Cohen S. Prenatal and postnatal hormone effects on the human brain and cognition. Eur J Physiol. 2013;465:557-71. doi: 10.1007/s00424-013-1268-2

[46] Kanai R, Rees G. The structural basis of interindividual differences in human behaviour and cognition. Nat Rev Neurosci. 2011;12:231-42. doi:10.1038/nrn3000

[47] Bastin C, Yakushev I, Bahri MA, Fellgiebel A, Eustache F, et al Cognitive reserve impacts on interindividual variability in resting-state cerebral metabolism in normal aging. Neuroimage. 2012;63:713-22. doi:10.1016/j.neuroimage.2012.06.074

[48] Tucker AM, Stern Y. Cognitive reserve in aging. Curr Alzheimer Res. 2011;8:354-60. 


\section{Competing Interests}

The authors declare no competing interests.

\section{Publishing Notes}

(C) 2014 Zihl et al. This work has been published open access under Creative Commons Attribution License CC BY 4.0, which permits unrestricted use, distribution, and reproduction in any medium, provided the original work is properly cited. Conditions, terms of use and publishing policy can be found at www.scienceopen.com.
Please note that this article may not have been peer reviewed yet and is under continuous post-publication peer review. For the current reviewing status please click here or scan the QR code on the right.

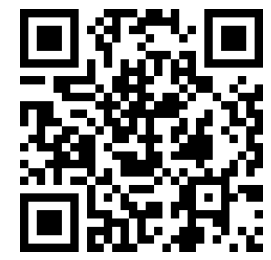

